\title{
Observation of $v_{\mu} \rightarrow v_{\tau}$ oscillations in the CNGS beam with the OPERA experiment
}

\author{
M. De Serio ${ }^{1, a}$ on behalf of the OPERA Collaboration \\ ${ }^{1}$ University of Bari \& I.N.F.N. Bari, I-70126 Bari, Italy
}

\begin{abstract}
The OPERA long-baseline neutrino experiment was exposed to the CNGS $v_{\mu}$ beam from 2008 to 2012, collecting 19505 interactions in the target. The evidence for oscillated $v_{\tau}$ appearance, based on the observation of three $v_{\tau}$ candidate events, has been previously reported. A fourth candidate event has been recently found in an extended data sample, corresponding to about $89 \%$ of the final statistics. The absence of a signal from $v_{\mu} \rightarrow v_{\tau}$ oscillations is currently excluded at $4.2 \sigma$. The status of the analysis is described in detail with special emphasis on the procedures applied for the selection of signal candidate events and the assessment of efficiencies and background. The fourth $v_{\tau}$ candidate event is presented and the significance of the observation is discussed.
\end{abstract}

\section{Introduction}

Neutrino oscillations in the atmospheric sector, first estabilished in 1998 by Super-Kamiokande [1] and later confirmed by several experiments (e.g. [2-4]), have been so far mainly investigated in disappearance mode, namely through the observation of a suppression of the measured $v_{\mu}$ flux with respect to the expectations.

The OPERA (Oscillation Project with Emulsion tRacking Apparatus) long-baseline neutrino experiment [5] was conceived to provide the first measurement of $v_{\mu} \rightarrow v_{\tau}$ oscillations at the atmospheric scale in direct appearance mode, i.e. through the detection of the $\tau$ leptons produced in charged current (CC) $v_{\tau}$ interactions. The detector, consisting of a modular target made of lead nuclear emulsion units complemented by electronic trackers and muon spectrometers, is located in the underground Gran Sasso Laboratory (LNGS, Italy) and was operated in the CNGS (CERN Neutrinos to Gran Sasso) $v_{\mu}$ beam [6] from 2008 to 2012 , collecting $17.97 \times 10^{19}$ protons on target.

\section{The OPERA experiment}

\subsection{CNGS beam}

The CNGS beam is a conventional wide-band $v_{\mu}$ beam produced at the CERN-SPS. Protons of $400 \mathrm{GeV} / \mathrm{c}$ hit a graphite target. The kaons and pions produced among secondary particles are selected in sign and momentum by means of two magnetic lenses (the horn and the reflector) and focused in a $1 \mathrm{~km}$ long decay tunnel pointing towards LNGS.

\footnotetext{
a e-mail: Marilisa.Deserio@ba.infn.it
} 
Table 1. CNGS beam performance. For each physics run, the number of neutrino interactions in the target is also reported.

\begin{tabular}{ccccc}
\hline Run & p.o.t. $\left(10^{19}\right)$ & SPS efficiency & Beam days & $v$ interactions \\
\hline 2008 & 1.70 & $61 \%$ & 123 & 1931 \\
\hline 2009 & 3.53 & $73 \%$ & 155 & 4005 \\
\hline 2010 & 4.09 & $80 \%$ & 187 & 4515 \\
\hline 2011 & 4.75 & $79 \%$ & 243 & 5131 \\
\hline 2012 & 3.86 & $82 \%$ & 257 & 3923 \\
\hline Total & 17.97 & $77 \%$ & 965 & 19505 \\
\hline
\end{tabular}

The beam energy profile has been optimised to observe the appearance of oscillated $v_{\tau}$ 's at LNGS, at a distance of $730 \mathrm{~km}$ from the neutrino source. The average energy $(\sim 17 \mathrm{GeV})$ is well above the threshold of the $v_{\tau}$ charged current interaction process and the prompt $v_{\tau}$ contamination from the decay of $D_{s}$ mesons is negligeable.

The CNGS beam performance over the five physics runs of the experiment is summarised in table 1 . The number of $v$ interactions recorded in the OPERA target is also reported.

\subsection{Detector}

The OPERA detector [7] is made of two identical super-modules, each one consisting of a target section followed by a muon spectrometer.

The target, with a total mass of $1.25 \mathrm{kt}$, has a modular structure and is segmented into 150000 basic units called bricks. A brick consists of 57 thin nuclear emulsion films interleaved with $561 \mathrm{~mm}$-thick lead plates with transverse sizes of $12.8 \times 10.2 \mathrm{~cm}^{2}$. Its dimension along the beam direction is $7.9 \mathrm{~cm}$, corresponding to about 10 radiation lengths.

Thanks to the excellent spatial resolution of nuclear emulsions $(\lesssim 1 \mu \mathrm{m})$, each brick acts as a stand-alone detector, allowing a precise three-dimensional reconstruction of the neutrino interaction vertex, as well as of the decay points of short-lived particles (e.g. the $\tau$ lepton). Moreover, the interleaving of emulsion films and plates of dense passive material (the so-called Emulsion Cloud Chamber tecnique) makes it possible to measure the momenta of charged particles through Multiple Coulomb Scattering [8] and identify electromagnetic showers initiated by $\gamma$ 's and $\pi^{0}$ 's.

Bricks are arranged in planar structures, called walls, with transverse dimensions of $\sim 6.7 \times 6.7 \mathrm{~m}^{2}$. Each wall, able to host $64 \times 52$ bricks, is coupled to a pair of tracker planes (TT), made of plastic scintillator strips of $2.6 \mathrm{~cm}$ width. TT planes have the primary tasks of providing the event trigger signal and identifying in real-time the brick containing the $v$ interaction. A doublet of special emulsion films, called Changeable Sheets (CS, [9]), glued on the downstream face of each brick, acts as an interface between the electronic tracker with a spatial accuracy of $1 \mathrm{~cm}$ and the emulsion/lead target with sub-micrometric resolution.

The spectrometer of each super-module is a dipolar magnet $\left(\sim 8.75 \times 8 \mathrm{~m}^{2}\right)$ made of two magnetized iron walls producing a field of $1.53 \mathrm{~T}$ with vertical lines of opposite directions in the two walls. The magnet is instrumented with planes of bakelite RPC's, inserted between the iron slabs of each wall to measure the range of stopping particles and track penetrating muons. The muon charge and momentum are measured by planes of drift tubes with a spatial resolution of $\sim 300 \mu \mathrm{m}$, placed in front, behind and in between the magnet walls . 


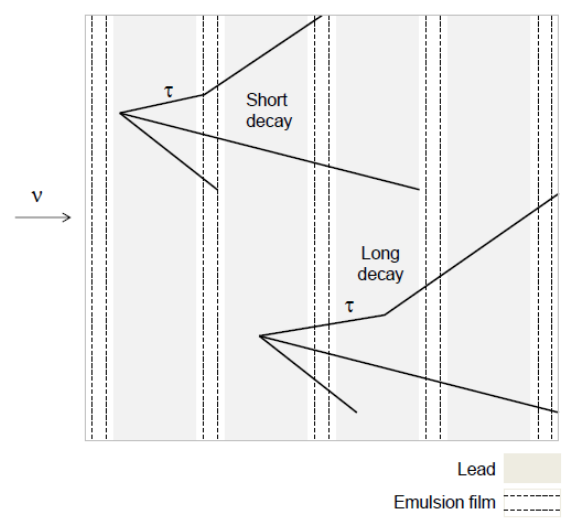

Figure 1. Detectable $\tau$ decay topologies: the decay is defined as short or long depending on whether it occurs in the same lead plate where the neutrino interacts or downstream of it.

The achieved muon momentum resolution is better than $20 \%$ in the relevant kinematical range ( $\lesssim 50 \mathrm{GeV} / \mathrm{c}$ ). The muon identification efficiency, as obtained by a combined analysis of spectrometer and scintillator tracker data, is $\sim 95 \%$.

A veto system, consisting of planes of glass RPC's, is placed in front of the first super-module to tag the interactions originating in the upstream rock.

\subsection{Event location and analysis}

The OPERA DAQ system was designed to run in a continuous triggerless mode. The detector remained sensitive during the inter-spill time and events not correlated with the beam (e.g. cosmic-ray muons) were used for monitoring purposes.

The synchronisation between the detector and the CNGS beam was performed offline through GPS with an accuracy of $100 \mathrm{~ns}$. A $10 \mathrm{~ns}$ clock, synchronised with the local GPS, was distributed to all sub-detectors. The event building was obtained by sorting in time the hits registered in a window of $3 \mu \mathrm{s}$.

Each reconstructed event typically extends across several bricks. A software algorithm computes a 3D map assigning a probability to contain the neutrino interaction vertex to each of the interested bricks. The most probable one is removed from the target, the corresponding CS doublet is detached and its films are analysed by means of high-speed automated microscopes ([10],[11]) to search for tracks compatible with the electronic detector hits.

If the search is successful, the brick is disassembled and the emulsion films are developed and sent to a network of scanning laboratories in Europe and in Japan for event location and analysis [12]. The tracks found in the CS are extrapolated to the most downstream film of the brick and followed back until they are not found in five consecutive films. The most upstream track disappearance point is tagged as candidate neutrino vertex point. In order to confirm and fully reconstruct the interaction, a surface of $1 \mathrm{~cm}^{2}$ is measured in 5 films upstream and 10 films downstream of the assumed vertex point. Sub-micrometric accuracy is achieved in the reconstruction of tracks thanks to the exposure of each brick to an optimal flux of high energy cosmic rays before disassembly [13]. A complex procedure [14] is then applied to search for possible decays of $\tau$ leptons produced in CC interactions 


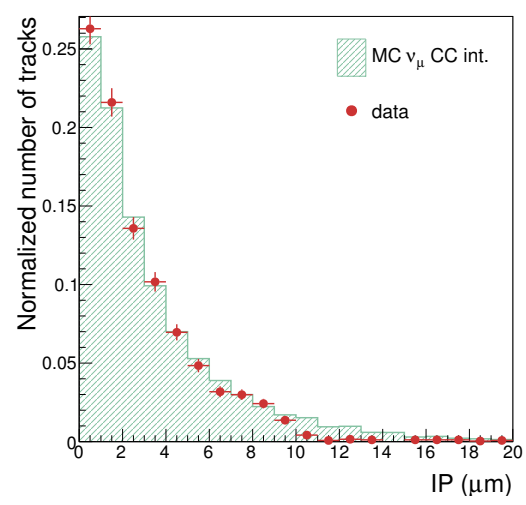

Figure 2. Track impact parameter distribution for a sample of located $v_{\mu} \mathrm{CC}$ interactions, compared to MC expectations.

of $v_{\tau}$ 's coming from the flavor conversion of CNGS $v_{\mu}$ 's. As shown in figure 1, two distinct topologies can occur, depending on whether the $\tau$ particle decays in the same lead plate where the neutrino interacts (short decays) or downstream of it (long decays). In both cases, daughter particles can be detected as tracks with large impact parameter with respect to the neutrino vertex. Figure 2 shows the distribution of the track impact parameter with respect to the reconstructed neutrino vertex for a sample of located $v_{\mu} \mathrm{CC}$ interactions, compared to Monte Carlo expectations. The distribution has an average value of about $3 \mu \mathrm{m}$ with tails mainly due to low energy particles. Any detected track in a fiducial volume around the neutrino vertex, showing an impact parameter larger than a threshold value depending on the distance from the interaction point, is thus further investigated to assess its nature. A full kinematical analysis is finally required for candidate events with a topology compatible with the production and decay of a $\tau$ lepton.

If the event location is not successful in the most probable brick, additional target units can be extracted and analysed according to the probability map, as it was done in runs 2008 and 2009.

The dependence of the location efficiency on the energy released in the TT is shown in figure 3 for a control sample of data from 2008 and 2009 runs. The first two bricks in the probability map were analysed for each event. Monte Carlo expectations are also shown. The comparison is made separately for events with $(1 \mu)$ and without $(0 \mu)$ a reconstructed muon in the final state [15]. As expected, a clear correlation can be seen for the $0 \mu$ sample, where the hadronic activity plays a crucial role in the location. The efficiency for the $1 \mu$ sample is almost flat, the event location being driven by the reconstruction of the muon track.

\section{$3 v_{\mu} \rightarrow v_{\tau}$ oscillation analysis}

The data set used in the present analysis consists of the whole sample of $0 \mu$ events collected during the 5 years of run and the $1 \mu$ events with a muon momentum smaller than $15 \mathrm{GeV} / \mathrm{c}^{1}$. For runs 2008 and 2009, all events have been searched for in the two most probable bricks, while only the first brick

\footnotetext{
${ }^{1}$ This kinematical cut improves the signal to noise ratio for the $v_{\tau}$ search with a negligible signal loss and a significant reduction $(\sim 25 \%)$ of the $v_{\mu}$-induced interaction sample.
} 


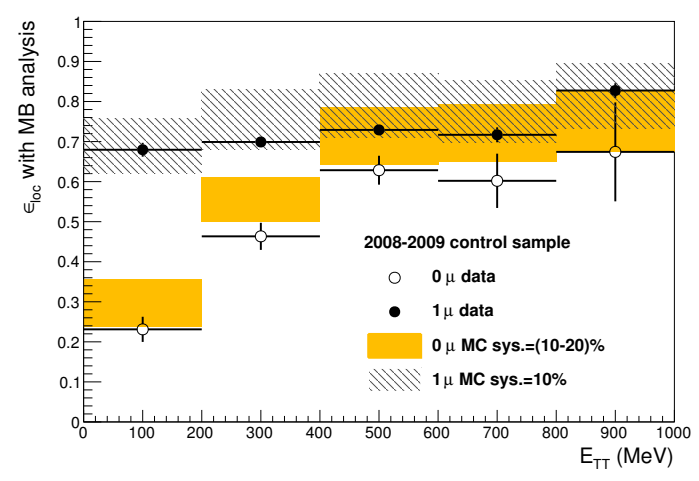

Figure 3. Location efficiency as a function of the energy deposited in the TT for a control sample of data collected in 2008 and 2009 runs (bullets), compared with MC (histograms). Error bars and bands represent the statistical and systematical uncertainties in the data and in the MC, respectively.

in the probability map has been analysed so far for runs $2010-2012$. The numbers of events are summarised in table 2.

Out of 4685 located neutrino interactions, four $v_{\tau}$ candidate events satisfying the selection criteria described in [15] have been observed. The first three events have been reported in detail [15-17]. Figure 4 shows a display of the fourth candidate event [18] as reconstructed in the brick, in the view transverse to the neutrino direction. The event was located in the second super-module, ten walls upstream of the spectrometer. The $v$ vertex, indicated as "v1" in the figure, consists of four tracks. One of them (track 1) exhibits a kink topology (decay vertex "v2") after $1090 \mu \mathrm{m}$. The measured kink angle is $137 \mathrm{mrad}$. The decay daughter particle has a momentum of $6.0_{-1.2}^{+2.2} \mathrm{GeV} / \mathrm{c}$ and shows an impact parameter with respect to the neutrino vertex of $146 \mu \mathrm{m}$. The track was followed in the downstream bricks and was found to stop in the spectrometer after leaving a signal in three RPC planes. The tracks 2, 3 and 4 were followed downstream as well, in order to exclude the presence of a muon or an electron at the neutrino vertex. In total, 20 bricks were analysed. Track 4 was classified as a proton, tracks 2 and 3 were identified as hadrons. Two electromagnetic showers initiated by the conversion of $\gamma$ 's and pointing to the neutrino vertex were also detected, as it can be seen in figure 4 .

Figure 5 shows the Monte Carlo distributions of the kinematical variables used to select candidate events in the $\tau$ decay channel into one hadron $(\tau \rightarrow 1 h): \theta_{\text {kink }}$ is the kink angle, $z_{\text {dec }}$ is the flight length, $p_{2 r y}$ is the daughter particle momentum, $p_{T}^{2 r y}$ is the transverse momentum at the decay vertex, $p_{T}^{\text {miss }}$ is the missing transverse momentum at the neutrino vertex, $\Delta \phi_{\tau H}$ is the angle between the $\tau$ lepton direction and the sum of the transverse momenta of the other particles produced in the interaction. Red vertical lines and bands represent the measured values of the above quantities for the fourth candidate event and the corresponding errors, respectively. Gray areas mark the regions excluded by the selection criteria. The measured values, reported in table 3 together with the applied cuts, are well within the expected signal region.

The estimated numbers of signal and background events for the analysed sample are summarised in table 4. In total, assuming $\Delta m_{23}^{2}=2.32 \mathrm{eV}^{2}$ and maximal mixing, $2.11 \pm 0.42 v_{\tau}$ events are expected with an estimated background of $0.233 \pm 0.041$ events. Given the detection of four candidate events, the observation of $v_{\mu} \rightarrow v_{\tau}$ oscillation in appearance mode is thus estabilished at $4.2 \sigma$ level. The number of observed $v_{\tau}$ events can be also used to compute the $90 \%$ confidence interval for $\Delta m_{23}^{2}$. 
Table 2. Numbers of events used for the $v_{\mu} \rightarrow v_{\tau}$ oscillation analysis.

\begin{tabular}{lcccccc}
\hline Run & 2008 & 2009 & 2010 & 2011 & 2012 & Total \\
\hline $0 \mu$ events & 148 & 250 & 209 & 223 & 149 & 979 \\
\hline $1 \mu$ events $\left(p_{\mu} \leq 15 \mathrm{GeV} / \mathrm{c}\right)$ & 534 & 1019 & 814 & 749 & 590 & 3706 \\
\hline Total number of events & 682 & 1269 & 1023 & 972 & 739 & 4685 \\
\hline
\end{tabular}

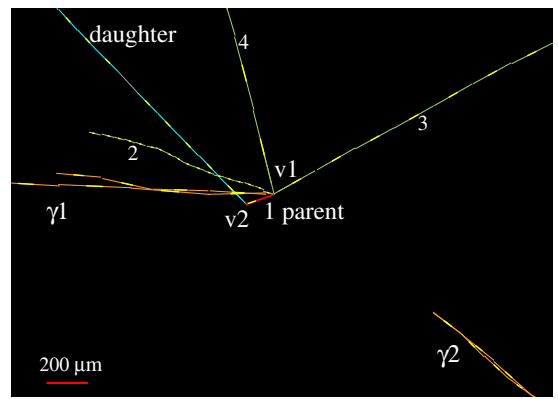

Figure 4. Display of the fourth candidate $v_{\tau}$ event in the view transverse to the neutrino direction.

Table 3. Measured values of the kinematical variables used to select candidate events in the $\tau \rightarrow 1 h$ decay channel for the fourth $v_{\tau}$ event and corresponding selection cuts.

\begin{tabular}{lcc}
\hline Variable & Measured value & Selection cut \\
\hline$\theta_{\text {kink }}(\mathrm{mrad})$ & $137 \pm 4$ & $>20$ \\
\hline$z_{\text {dec }}(\mu \mathrm{m})$ & $406 \pm 30$ & $<2600$ \\
\hline$p_{2 r y}(\mathrm{GeV} / \mathrm{c})$ & $6.0_{-1.2}^{+2.2}$ & $>2$ \\
\hline$p_{T}^{2 r y}(\mathrm{GeV} / \mathrm{c})$ & $0.82_{-0.16}^{+0.30}$ & $>0.6$ \\
\hline$p_{T}^{\text {miss }}(\mathrm{GeV} / \mathrm{c})$ & $0.55_{-0.20}^{+0.30}$ & $<1$ \\
\hline$\Delta \phi_{\tau H}($ degrees $)$ & $166_{-31}^{+2}$ & $>90$ \\
\hline
\end{tabular}

Table 4. Estimated signal and background events for the analysed sample. Numbers are reported separately for each of the four $\tau$ decay channels. Observed events in each channel are also reported.

\begin{tabular}{cccc}
\hline $\begin{array}{c}\text { Decay } \\
\text { channel }\end{array}$ & $\begin{array}{c}\text { Expected signal } \\
\left(\Delta m_{23}^{2}=2.32 \mathrm{eV}^{2}\right)\end{array}$ & $\begin{array}{c}\text { Total } \\
\text { background }\end{array}$ & $\begin{array}{c}\text { Observed } \\
\text { events }\end{array}$ \\
\hline$\tau \rightarrow h$ & $0.41 \pm 0.08$ & $0.033 \pm 0.006$ & 2 \\
\hline$\tau \rightarrow 3 h$ & $0.57 \pm 0.11$ & $0.155 \pm 0.030$ & 1 \\
\hline$\tau \rightarrow \mu$ & $0.52 \pm 0.10$ & $0.018 \pm 0.007$ & 1 \\
\hline$\tau \rightarrow e$ & $0.62 \pm 0.12$ & $0.027 \pm 0.005$ & 0 \\
\hline Total & $2.11 \pm 0.42$ & $0.233 \pm 0.041$ & 4 \\
\hline
\end{tabular}

The estimated interval using the Feldman - Cousins method [19] is $[1.8,5.0] \times 10^{-3} \mathrm{eV}^{2}$. A similar result is obtained employing a Bayesian approach [20] with a flat prior. 

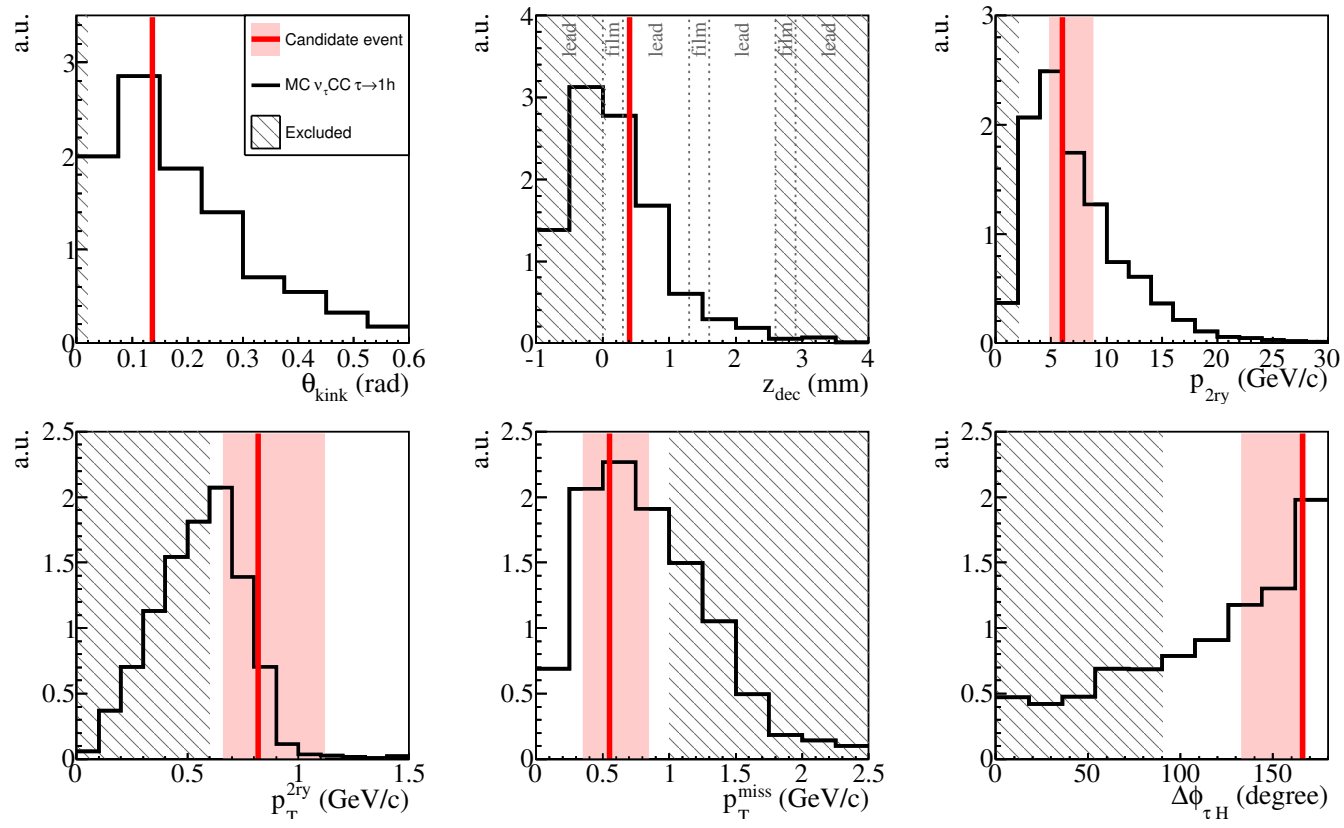

Figure 5. MC distributions of the kinematical variables used to select candidate events in the $\tau \rightarrow 1 h$ decay channel.

\section{Conclusions}

After a successful data taking in the CNGS beam from 2008 to 2012, the OPERA experiment has recently established the observation of $v_{\mu} \rightarrow v_{\tau}$ oscillations in the atmospheric sector at $4.2 \sigma$ level. The result is based on the detection of four $v_{\tau}$ candidate events in a sample of 4685 located neutrino interactions. The $v_{\tau}$ search is currently being applied to a larger sample including events located in the second and third bricks of the probability map.

\section{References}

[1] Y. Fukuda et al. (Super-Kamiokande), Phys. Rev. Lett. 811562 (1998);

J. Hosaka et al. (Super-Kamiokande), Phys. Rev. D 74032002 (2006)

[2] M. Ambrosio et al. (MACRO), Phys. Lett. B 434451 (1998)

[3] M. H. Ahn, et al. (K2K), Phys. Rev. D 74072003 (2006)

[4] P. Adamson et al. (MINOS), Phys. Rev. Lett. 108191801 (2012)

[5] M. Guler et al. (OPERA), CERN-SPSC-2000-028

[6] CNGS project, http://proj-cngs.web.cern.ch/proj-cngs

[7] R. Acquafredda et al. (OPERA), J. Instrum. 3 P04018 (2009)

[8] N. Agafonova et al. (OPERA), New J. Phys. 14013026 (2012)

[9] A. Anokhina et al. (OPERA), J. Instrum. 3 P07005 (2008)

[10] K. Morishima, T. Nakano, J. Instrum. 5 P04011 (2010) 
[11] N. Armenise et al., Nucl. Instr. Meth. A 551261 (2005);

L. Arrabito et al., Nucl. Instr. Meth. A 568578 (2006)

[12] N. Agafonova et al. (OPERA), J. Instrum. 4 P06020 (2009)

[13] E. Barbuto et al., Nucl. Instrum. Methods A 525485 (2004)

[14] N. Agafonova et al. (OPERA), Eur. Phys. J. C 742986 (2014)

[15] N. Agafonova et al. (OPERA), J. High Energy Phys. 11036 (2013)

[16] N. Agafonova et al. (OPERA), Phys. Lett. B 691138 (2010)

[17] N. Agafonova et al. (OPERA), Phys. Rev. D 89051102 (R) (2014)

[18] N. Agafonova et al. (OPERA), Prog. Theor. Exp. Phys. 101C01 (2014)

[19] G. J. Feldman and R. D. Cousins, Phys. Rev. D 573873 (1998)

[20] J. Beringer et al. (Particle Data Group), Phys. Rev. D 86010001 (2012) 